\title{
Adaptação Acadêmica de Estudantes de Primeiro Ano de Psicologia
}

\author{
Adriana Benevides Soares* \\ Universidade Salgado de Oliveira - Universo, Niterói, RJ, Brasil \\ ORCID: https://orcid.org/0000-0001-8057-6824 \\ Claudio de Almeida Lima** \\ Universidade Salgado de Oliveira - Universo, Niterói, RJ, Brasil \\ ORCID: https://orcid.org/0000-0002-5404-7029 \\ Marcia Cristina Monteiro*** \\ Universidade Salgado de Oliveira - Universo, Niterói, RJ, Brasil \\ ORCID: https://orcid.org/0000-0003-3455-2690 \\ Bruno de Alcântara Sousa***** \\ Universidade Salgado de Oliveira - Universo, Niterói, RJ, Brasil \\ ORCID: https://orcid.org/0000-0002-2063-7282 \\ Giselle Glória Balbino dos Santos****** \\ Universidade Salgado de Oliveira - Universo, Niterói, RJ, Brasil \\ ORCID: https://orcid.org/0000-0001-6738-3059 \\ Isabela da Silva Rodrigues $* * * * * * *$ \\ Universidade Salgado de Oliveira - Universo, Niterói, RJ, Brasil \\ ORCID: https://orcid.org/0000-0002-3263-5575
}

\section{RESUMO}

O objetivo do estudo foi verificar se as variáveis expectativas acadêmicas, satisfação com a experiência acadêmica, maturidade para a escolha profissional e habilidades sociais (HS) explicam a variável critério adaptação acadêmica. Participaram 212 universitários de primeiro ano do curso de Psicologia. Utilizou-se os seguintes instrumentos: Questionário de Vivências Acadêmicas (QVA-r), Escala de Expectativas Acadêmicas de Estudantes Ingressantes na Educação Superior (EEAEIES), Escala de Satisfação com a Experiência Acadêmica (ESEA), Inventário de Habilidades Sociais (IHS) e Escala de Maturidade para Escolha Profissional (EMEP). Utilizou-se para a análise dos dados a Regressão Linear Múltipla. Constatou-se que as expectativas acadêmicas, a satisfação com a experiência acadêmica e as HS impactaram positivamente nas vivências acadêmicas. Houve impacto positivo dos fatores Curso da ESEA e Autoconhecimento da EMEP e impacto negativo do fator Determinação. O presente estudo contribuiu principalmente mostrando a importância do comportamento das variáveis do modelo testado. Por ser a adaptação acadêmica um construto multideterminado, os resultados alcançados podem apontar para ações de intervenções no acolhimento de universitários ingressantes, considerando aspectos como autoconhecimento, determinação, expectativas acadêmicas, conhecimento da realidade e satisfação com a graduação.

Palavras-chave: estudantes universitários, adaptação acadêmica, psicologia. 


\title{
Academic Adaptation of the First Year of Psychology Students
}

\begin{abstract}
The aim of the study was to verify if the variables academic expectations, satisfaction with academic experience, maturity for professional choice and social skills explain the variable academic adaptation criterion. Participated 212 university students in the 1st year of the Psychology course. The following instruments were used: Academic Experience Questionnaire (AEQ), Academic Expectations Scale for Students in Higher Education (AESSHE), Academic Experience Satisfaction Scale (AESS), Social Skills Inventory (SSI) and Scale of Maturity for the Professional Election (SMPE). Multiple Linear Regression was used for data analysis. It was found that the academic expectations, satisfaction with the academic experience and the social skill impact positively on the academic experiences. There was a positive impact on the Factors of the AESS and Self-knowledge of the SMPE and negative impact of the factor Determination. The present study contributed mainly by showing the importance of the behavior of the variables of the tested model. Once academic adaptation is a multidetermined construct, the results achieved can point to interventions in the reception of university students, considering aspects such as self-knowledge, determination, academic expectations, knowledge of reality and satisfaction with undergraduate education.
\end{abstract}

Keywords: university students, academic adaptation, psychology.

\section{Adaptación Académica de Estudiantes de Primer Año de Psicología}

\section{RESUMEN}

El objetivo del estudio fue verificar si las variables expectativas académicas, satisfacción con la experiencia académica, madurez para la elección profesional y habilidades sociales explican el criterio variable de adaptación académica. Participaron 212 universitarios del $1^{\circ}$ año del curso de Psicología. Se utilizaron los siguientes instrumentos: Cuestionario de Vivencias Académicas (QVA), Escala de Expectativas Académicas de Estudiantes Ingresos en la Educación Superior (EEAEIES), Escala de Satisfacción con la Experiencia Académica (ESEA), Inventario de Habilidades Sociales (IHS) y Escala de Madurez para la Elección Profesional (EMEP). Se utilizó para el análisis de los datos la Regresión Lineal Múltiple. Se constató que las expectativas académicas, satisfacción con la experiencia académica y las HS han impactado positivamente en las vivencias académicas. Hubo impacto positivo en los factores Curso de la ESEA y Autoconocimiento de la EMEP e impacto negativo del factor Determinación. El presente estudio contribuyó principalmente al mostrar la importancia del comportamiento de las variables del modelo probado. Debido a que la adaptación académica es una construcción multideterminada, los resultados logrados pueden apuntar a intervenciones en la recepción de estudiantes universitarios, considerando aspectos como el autoconocimiento, la determinación, las expectativas académicas, el conocimiento de la realidad y la satisfacción con la graduación.

Palabras clave: estudiantes universitarios, adaptación académica, psicologia. 
O Ensino Superior é caracterizado pela multiplicidade de situações psicossociais experimentadas pelo estudante, podendo ser considerado um contexto privilegiado para o desenvolvimento do discente (Oliveira, Merino, Privado, \& Almeida, 2017). O aluno tem a oportunidade de ampliar a rede de relacionamentos, aprender a administrar diferentes compromissos e tarefas, lidar com figuras de autoridade, entre outras situações. Entretanto, para muitos estudantes o primeiro ano também é um momento que pode ser compreendido como de difícil manejo pelos inúmeros desafios a serem gerenciados (Soares et al, 2018), podendo levar ao abandono ou a interrupção do curso, ou até ao desenvolvimento/agravamento de problemas de saúde mental. Soares, Almeida, Diniz e Guisande (2006), no Modelo Multidimensional de Ajustamento de jovens ao contexto Universitário - MMAU, estabelecem a importância de diferentes variáveis para explicar a adaptação acadêmica. A adaptação acadêmica pode ser compreendida por meio do comprometimento do aluno com o curso, com a capacidade de estabelecer novos vínculos, com a presença ou ausência de estresse e ansiedade diante das demandas que favorecerão a permanência do estudante na instituição, o processo de formação profissional e para a vida (Oliveira et al., 2017). Soares et al. (2006) consideram que as características dos estudantes, como idade, sexo, classe econômica, experiência acadêmica e aspectos comportamentais e cognitivos, relacionam-se direta ou indiretamente às características acadêmicas, como médias obtidas para ingresso e opção de curso. Pesquisas (Fernandéz, Araújo, Vacas, Almeida e González, 2017; Oliveira \& Morais, 2015) apontam algumas adversidades encontradas na universidade que implicam na adaptação do estudante ao Ensino Superior.

Ademais, a graduação em Psicologia envolve o contato com o outro e com os conteúdos acadêmicos diretamente relacionados com a subjetividade humana, o que inicialmente pode ser um aspecto desafiador para muitos estudantes que realizaram a escolha pelo curso de forma incipiente. Em estudo realizado por Oliveira e Morais (2015), os autores investigaram as principais dificuldades de adaptação acadêmica de estudantes de instituição pública no curso de Engenharia e considerando os elevados índices de evasão. Os resultados apontaram para as dimensões pessoal e estudo do QVA-r e com menores índices para as dimensões carreira, interpessoal e institucional. Matta, Lebrão e Heleno (2017) realizaram estudo de revisão de artigos entre os anos de 2005 a 2015 referentes à adaptação acadêmica incluindo as variáveis vivências acadêmicas, rendimento e evasão. O estudo apontou para o fato de que os relacionamentos interpessoais podem favorecer o rendimento acadêmico e adiar a decisão de abandono da Educação Superior. 
Fernandéz et al. (2017) ressaltam que a adaptação acadêmica não é uma experiência invariável, mas uma multiplicidade de vivências complexas. Em estudo com universitários espanhóis de cursos de Ciências, Ciências Sociais, Direito, Humanidades e Ciências da Saúde e Engenharia, os pesquisadores apontaram que as características de ingresso do aluno interagem com variáveis de processo e fatores institucionais e devem ser considerados os aspectos culturais nos quais as experiências são produzidas. Nesta perspectiva, compreende-se que a adaptação à universidade não é um bloco único, mas é constituída de diferentes aspectos. Ademais, compreender como diferentes variáveis se comportam na adaptação do estudante ao curso de Psicologia pode contribuir na qualidade da formação discente (Travassos \& Mourão, 2018) e em ações melhor direcionadas de apoio ao aluno. A adaptação acadêmica é um construto multideterminado, com vários componentes que podem regular o comportamento (Freitas, Raposo, \& Almeida, 2007) e precisam apresentar construtos claramente definidos, que especifiquem a pertinência dos componentes propostos e sua relação para a vida acadêmica. Assim, as variáveis que constituem o modelo a ser testado de adaptação acadêmica do estudante de Psicologia são as expectativas acadêmicas, a maturidade para escolha profissional e as habilidades sociais.

Neste contexto, a expectativa acadêmica é uma das variáveis que se apresenta como mediadora da adaptação à Educação Superior (Araujo \& Almeida 2015). Os estudos buscam avaliar o quanto as expectativas no início da universidade se mantêm no decorrer do curso, o quanto se aproximam da realidade e o quanto afetam a qualidade de ajustamento do estudante no Ensino Superior (Araújo \& Almeida, 2015; Bisinoto, Rabelo, Marinho-Araújo, \& Fleith, 2016; Soares et al, 2018).

As expectativas são crenças sobre o que os estudantes esperam encontrar na vida acadêmica, e são constituídas por aspectos pessoais e contextuais anteriores ao ingresso na universidade (Soares et al, 2018). Essas expectativas vão intervir nas vivências dos estudantes, principalmente no primeiro ano, e podem favorecer o ajustamento nesse novo contexto (Araújo \& Almeida, 2015). Aqueles que possuem expectativas mais próximas da realidade irão transitar por esse período com melhores níveis de ajustamento, diferente dos estudantes que apresentam expectativas irrealistas, subestimando os desafios e demandas que irão enfrentar na universidade (Araújo \& Almeida, 2015).

Neste sentido, o estudo realizado por Bisinoto et al. (2016), que contou com uma amostra de 6.913 estudantes de uma universidade federal brasileira, teve como objetivo compreender e mapear as expectativas que os alunos trazem ao ingressar no Ensino Superior. Os resultados mostraram que as maiores expectativas estavam relacionadas à formação 
acadêmica de qualidade (aprofundamento do conhecimento), ao apoio recebido de professores no processo de aprendizagem e ao preparo para a prática profissional, como também esperavam se tornar mais autônomos e responsáveis. Em sequência de importância apontada pelos alunos, a perspectiva de sucesso profissional, oportunidade de internacionalização e intercâmbio, compromisso social e acadêmico, ampliação das relações interpessoais e, por fim, aparece como menos expressiva a preocupação com a autoimagem (expectativas de familiares e amigos em relação ao ingresso na universidade). Também foi observado que as mulheres apresentam maiores expectativas em todas essas dimensões avaliadas.

Outrossim, a variável satisfação acadêmica é apontada pela literatura (Santos, Polydoro, Scortegagna e Linden, 2013; Schleich, Polydoro, \& Santos, 2006; Suehiro \& Andrade, 2018) como de relevância para a adaptação do estudante à Educação Superior. O termo "satisfação acadêmica" tem como referência a qualidade da aquisição de conhecimentos por parte dos estudantes e como estes avaliam estes conteúdos, mediante um processo dinâmico perpassado por variáveis institucionais e pessoais (Santos et al., 2013). A experiência que o estudante obtém na relação com a sua formação, em aspectos que se vinculam com a qualidade da instituição, dos professores, do curso, são elementos que compõem o conjunto do termo satisfação acadêmica (Schleich et al., 2006). Para esses autores há três fatores importantes que envolvem a satisfação acadêmica: a satisfação com o curso, a oportunidade de desenvolvimento e a satisfação com a instituição.

Em um estudo realizado por Santos et al. (2013) com 203 estudantes do curso de Psicologia e Odontologia de uma universidade particular do Rio Grande do Sul, que teve como objetivo avaliar a integração e a satisfação acadêmica, foi possível encontrar um alto índice de satisfação com a vida acadêmica, o que, segundo os autores, é imprescindível para uma saudável vivência acadêmica. A dimensão institucional do Questionário de Vivências Acadêmicas - reduzido (QVA-r) correlacionou-se com todas as dimensões da Escala de Satisfação com a Experiência Acadêmica (ESEA), indicando a importância da dimensão institucional para uma boa adaptação, sendo que as pontuações mais elevadas se dão para os alunos ingressantes.

Ao descrever o percurso inicial dos estudantes universitários, Suehiro e Andrade (2018), em seu estudo com 232 estudantes de vários cursos de uma universidade publica federal do interior, teve como proposta identificar quais aspectos ou variáveis contribuem de forma efetiva na satisfação e, consequentemente, na tomada de decisão dos alunos do primeiro ano da graduação. Os resultados do estudo apontaram para uma maior satisfação com o curso e menor satisfação com a dimensão oportunidade de desenvolvimento, o que 
pode indicar prevalência das relações interpessoais como fator importante para a permanência no curso e, de forma inversa, quanto menor o investimento da instituição em atividades extracurriculares e mudanças curriculares, mais insatisfeitos se tornam os alunos.

Outra variável que compõe o modelo teórico proposto a ser testado é a maturidade para a escolha profissional. No modelo teórico de Neiva (2014) a maturidade para escolha profissional é definida a partir das dimensões: determinação (o quanto o indivíduo está definido e seguro com relação à escolha profissional); responsabilidade (o quanto o indivíduo está engajado no processo de escolha profissional e promove ações para tomar esta decisão); independência (como o indivíduo processa esta decisão de forma autonôma); autoconhecimento (o conhecimento que o indivíduo possui sobre vários aspectos de si mesmo importantes para a escolha profissional) e conhecimento da realidade (o conhecimento que o sujeito tem das instituições educativas, das profissões, do mercado de trabalho).

A escolha profissional tradicionalmente apresenta estreita relação com o período da adolescência (Frischenbruder, 1999; Magalhães, Lassance, \& Gomes, 1998; Osipow, 1999), sendo uma etapa do desenvolvimento caracterizada pela formação e pelos conflitos de identidade e vinculada a esta crise de identidade está a escolha profissonal (Moura \& Menezes, 2004; Neiva, 1995). Entretanto, a literatura aponta que a busca pela escolha de uma carreira não está restrita ao adolescente típico, mas ao jovem que já se encontra no Ensino Superior e por vocação errada, imaturidade e informações prévias insuficientes abandonam a graduação (Bardagi \& Hutz, 2010).

Lehman (2014) pesquisou a re-escolha em 180 estudantes de diferentes graduações de um serviço de orientação profissional em um período de cinco anos. O autor realizou a análise das causas das desistências e apontou a falta de elaboração de um processo de escolha inicial como um dos fatores da desistência. A pesquisa sinaliza a necessidade de desenvolvimento de políticas públicas em orientação profissional e o desenvolvimento de núcleos de atendimento aos alunos.

Lorga (2017) pesquisou a maturidade para escolha profissional em estudantes terceranistas do Ensino Médio. Participaram 200 alunos, sendo 100 de escola pública e 100 de particular, de ambos os sexos, com idade entre 16 e 19 anos. Concluiu-se que os participantes apresentaram maturidade satisfatória, sendo que os estudantes de escola pública obtiveram escores maiores do que aqueles de escola particular. A autora sinaliza ainda que, além da preocupação com as aprovações, os alunos também lidam com as incertezas do futuro profissional e com outros aspectos que podem interferir com a tomada de decisão. 
Esse novo contexto acadêmico da graduação em Psicologia requer do jovem estudante um repertório ligado as áreas do relacionamento interpessoal, rendimento acadêmico e do ajustamento emocional (Bartholomeu, Nunes, \& Machado, 2008). Considera-se a universidade como um local onde são geradas oportunidades de aprendizagens que vão além do conhecimento acadêmico, possibilitando o desenvolvimento das HS e da Competência Social (CS). O conceito de HS pode ser definido como "um construto descritivo dos comportamentos sociais valorizados em determinada cultura com alta probabilidade de resultados favoráveis para os indivíduo, seu grupo e comunidade que podem contribuir para um desempenho socialmente competente em tarefas interpessoais" (A. Del Prette \& Z. A. P. Del Prette, 2017a, p. 24) e CS como "um construto avaliativo do desempenho de um indivíduo (pensamentos, sentimentos e ações) em uma tarefa interpessoal que atende aos objetivos do indivíduo e às demandas da situação e cultura, produzindo resultados positivos conforme critérios e instrumentais e éticos" (A. Del Prette \& Z. A. P. Del Prette, 2017b, p. 37). Um repertório empobrecido de HS requesitadas em situações acadêmicas pode dificultar o desempenho acadêmico, ou até levar à evasão (Oliveira et al., 2017).

$\mathrm{Na}$ literatura, encontramos estudos empíricos que investigaram a relação de HS no contexto das vivências acadêmicas (Bolsoni-Silva, Loureiro, \& Oliveira, 2010). Bolsoni-Silva et al. (2010) desenvolveram estudo com objetivo de descrever as HS apresentadas por estudantes universitários ao longo dos anos de graduação. Buscou-se identificar as principais alterações ocorridas ao longo dos períodos, por meio da comparação entre os grupos de estudantes de diferentes anos $\left(1^{\circ}\right.$ e $2^{\circ}, 1^{\circ}$ e $3^{\circ}, 2^{\circ}$ e $\left.3^{\circ}\right)$ dos períodos integral e noturno e de homens e mulheres e à apresentação das análises funcionais das interações, observando os comportamentos e contextos mais recorrentes. A amostra contou com 85 estudantes universitários do curso de Desenho Industrial. As maiores diferenças encontraram-se nas comparações entre os anos, sugerindo que os dois primeiros anos requerem maiores investimentos em intervenções, pois apresentam maiores dificuldades em comportamentos de comunicação, expressividade e resolução de conflitos.

Diferentes correlações positivas entre fatores específicos de habilidades sociais e fatores pessoais e contextuais de vivências acadêmicas foram encontrados por Nogueira et al., (2020), evidenciando que um bom repertório de habilidades sociais associam-se a qualidade das vivências acadêmicas e ao desempenho acadêmico fomentando a adaptação à universidade. Diante do exposto em tela, o presente estudo tem como objetivo verificar se as variáveis expectativas acadêmicas, satisfação com a experiência acadêmica, maturidade para a 
escolha profissional e habilidades sociais explicam a variável critério adaptação acadêmica em estudantes do primeiro ano do curso de Psicologia.

\section{Método}

\section{Participantes}

Participaram 212 estudantes universitários de primeiro ano do curso de Psicologia com idades variando de 17 a 52 anos $(M=24,07$ e $\mathrm{DP}=7,94)$ sendo $45(21,6 \%)$ homens, 167 $(78,4 \%)$ mulheres; $165(77,9 \%)$ de IES privadas e $47(22,1 \%)$ de públicas; $13(6,5 \%)$ da classe social A, $10(4,7 \%)$ da B1, $77(36,2 \%)$ da B2, $66(31,0 \%)$ da C1, $36(16,9 \%)$ da C2, 10 $(4,7 \%)$ da D/E; $172(80,8 \%)$ solteiros, $30(14,6 \%)$ casados, 10 (4,7\%) declararam outros estados civis.

\section{Instrumentos}

Questionário de Vivências Acadêmicas (QVA-r) (Almeida, Ferreira \& Soares, 2002 adaptado por Granado, Santos, Soares, \& Guisande, 2005). É um instrumento composto por 55 itens distribuidos em cinco dimensões: Pessoal (14 itens, $\alpha=0,84$ ); Interpessoal (12 itens, $\alpha=0,82$ ); Carreira (14 itens, $\alpha=0,86$ ); Estudo (nove itens, $\alpha=0,78$ ) e Institucional (oito itens, $\alpha=0,77$ ).

Escala de Expectativas Acadêmicas de Estudantes Ingressantes na Educação Superior (EEAEIES) (Marinho-Araujo, Fleith, Almeida, Bisinoto, \& Rabelo, 2015). Composto de 61 itens distribuídos em 7 fatores: 1) Formação Acadêmica de Qualidade (12 itens, $\alpha=0,82$ ); 2) Compromisso Social e Acadêmico (13 itens, $\alpha=0,90)$; 3) Ampliação das Relações Interpessoais (oito itens, $\alpha=0,84$ ); 4) Oportunidade de Internacionalização e Intercâmbio (oito itens, $\alpha=0,87$ ); 5) Perspectiva de Sucesso Profissional (cinco itens, $\alpha=$ 0,71); 6) Preocupação com a Autoimagem (seis itens, $\alpha=0,78$ ) e 7) Desenvolvimento de Competências Transversais (nove itens, $\alpha=0,79$ ).

Escala de Satisfação com a Experiência Acadêmica (ESEA) (Schleich et al., 2006). É composta por 35 itens divididos em três fatores: 1) Satisfação com o curso (13 itens, $\alpha=$ 0,90); 2) Oportunidade de desenvolvimento (10 itens, $\alpha=0,87$ ) e 3) Satisfação com a instituição (12 itens, $\alpha=0,87$ ). 
Escala de Maturidade para a Escolha Profissional (EMEP) (Neiva, 2014). É constituída por 23 itens positivos (que indicam maturidade) e 22 negativos (que indicam imaturidade). Possui cinco subescalas: Determinação (10 itens, $\alpha=0,91$ ); Responsabilidade (10 itens, $(\alpha=0,75)$; Independência (oito itens, $\alpha=0,75$ ); Autoconhecimento (sete itens, $\alpha=$ 0,75) e Conhecimento da Realidade Profissional (10 itens, $\alpha=0,82$ ).

Inventário de Habilidades Sociais (IHS) (Z. A. P. Del Prette \& A. Del Prette, 2001). Composto por 38 itens, distribuído em cinco fatores: 1) Enfrentamento e Autoafirmação com risco (11 itens, $\alpha=0,96)$; 2) Autoafirmação na Expressão de Afeto Positivo (sete itens, $\alpha=0,86$ ); 3) Conversação e Desenvoltura Social (sete itens, $\alpha=0,81$ ); 4) Autoexposição a Desconhecidos ou a Situações Novas (quatro itens, $\alpha=0,75$ ) e 5) Autocontrole da Agressividade (três itens, $\alpha=0,74$ ). Na análise de consistência interna foi obtido coeficiente Alpha de Cronbach de 0,75.

\section{Procedimentos Éticos}

Este estudo foi aprovado no Comitê de Ética em pesquisa conforme o CAAE 66060117.6.0000.5289 em 16/03/2017. Os objetivos da pesquisa foram elucidados a todos os participantes que assinaram o Termo de Consentimento Livre e Esclarecido assegurando-se o sigilo de sua identidade e a possibilidade de retirar o seu assentimento de participação a qualquer momento.

\section{Procedimentos de Coleta de Dados}

Foi realizado contato com os docentes do primeiro período de Psicologia das Universidades para agendamento do dia e horário para aplicação dos questionários. Os inventários foram aplicados para grupos de aproximadamente 20 alunos de forma simultânea. Os participantes preencheram os cinco instrumentos respeitando a seguinte ordem: EEAEIES, ESEA, EMEP, IHS e QVA-r, sem nenhuma influência ou tempo definido para o preenchimento. Todos responderam o Questionário Sociodemográfico.

\section{Procedimentos de Análise de Dados}

Foi utilizada a Regressão Linear Múltipla (RLM) para verificar a relação entre as variáveis explicativas expectativas acadêmicas, satisfação com a experiência acadêmica, 
maturidade para escolha profissional, habilidades sociais e a variável critério vivências acadêmicas.

\section{Resultados}

Observa-se na Tabela 1 a RLM das variáveis explicativas nas dimensões da variável critério. Para a primeira dimensão houve impacto negativo do fator Determinação que explicou $29 \%$ da variável pessoal. Na segunda dimensão verificou-se impacto negativo dos fatores da EAEES: Formação Acadêmica de Qualidade, Compromisso Social e Acadêmico, Ampliação das Relações Interpessoais, Oportunidade de Internacionalização e Intercâmbio, Perspectiva de Sucesso Profissional, Preocupação com a Autoimagem e Desenvolvimento de Competências Transversais. Houve impacto positivo do fator Curso da ESEA. O conjunto de variaveis explicou $33 \%$ da variável critério interpessoal. A variável Curso da EAEES impactou positivamente na dimensão Carreira, explicando $32 \%$ da variável. O fator Oportunidade de Desenvolvimento da ESEA, o fator Autoexposição a Desconhecidos e Situações Novas do IHS e o fator Conhecimento da Realidade Profissional da EMEP impactaram positivamente na variável critério estudo. $\mathrm{O}$ conjunto dessas variáveis explicou 46\% da variável critério. O fator Determinação da EMEP apresentou impacto negativo e o Conhecimento da Realidade Profissional também da EMEP apresentou impacto positivo na variável critério Institucional. O conjunto de variáveis explicou $37 \%$ da variável critério Institucional. Para o escore geral das Vivências Acadêmicas identificou-se um impacto positivo no fator Curso da ESEA e do fator Autoconhecimento da EMEP e impacto negativo no fator Determinação. O conjunto de variáveis explicou $49 \%$ da variável critério vivências acadêmicas. 
Adriana Benevides Soares, Claudio de Almeida Lima, Marcia Cristina Monteiro, Bruno de Alcântara Sousa, Giselle Glória Balbino dos Santos, Isabela da Silva Rodrigues

\section{Tabela 1}

Regressão Linear Múltipla dos fatores das variáveis Expectativas Acadêmicas,

Satisfação com a Experiência Acadêmica, Habilidades Sociais e Maturidade para Escolha Profissional nas dimensões da Escala de Vivências Acadêmicas

\begin{tabular}{|c|c|c|c|c|}
\hline \multicolumn{5}{|c|}{ Dimensão Pessoal do QVA-r } \\
\hline R2ajustado $=0,29$ & \multicolumn{4}{|l|}{$\mathrm{F}=3,40 ; \mathrm{p}<0,001$} \\
\hline Escalas & Variáveis Explicativas & Beta & $t$ & Sig \\
\hline Mat_Esc_Prof & Determinação & $-0,23$ & $-3,06$ & $0,00 *$ \\
\hline \multicolumn{5}{|c|}{ Dimensão Interpes do QVA-r } \\
\hline R2ajustado $=0,33$ & \multicolumn{4}{|l|}{$\mathrm{F}=3,88 ; \mathrm{p}<0,001$} \\
\hline Escalas & Variáves Explicativas & Beta & $t$ & Sig \\
\hline \multirow{7}{*}{ Expec_Acad } & Form_Aca_Qua & $-0,84$ & $-2,21$ & $0,03^{*}$ \\
\hline & Com_Soc_Aca & $-0,93$ & $-2,61$ & $0,01^{*}$ \\
\hline & Ampl_Rel_Int & $-0,67$ & $-2,50$ & $0,01^{*}$ \\
\hline & Apo_Int_Inc & $-0,75$ & $-2,07$ & $0,04^{*}$ \\
\hline & Per_Suc_Pro & $-0,42$ & $-2,21$ & $0,03 *$ \\
\hline & Pre_Aut_Ima_ & $-0,48$ & $-2,02$ & $0,05^{*}$ \\
\hline & Com_Tran & $-1,21$ & $-2,21$ & $0,00 *$ \\
\hline Sat_Exp_Aca & Curso & 0,43 & 3,44 & $0,00 *$ \\
\hline \multicolumn{5}{|c|}{ Dimensão Carreira do QVA-r } \\
\hline R2ajustado $=0,46$ & \multicolumn{4}{|l|}{$F=6,13 ; p<0,001$} \\
\hline Escalas & Variáveis Explicativas & Beta & $t$ & Sig \\
\hline Sat_Exp_Acad & Curso & 0,31 & 4,33 & $0,00 *$ \\
\hline \multicolumn{5}{|c|}{ Dimensão Estudo do QVA-r } \\
\hline R2ajustado $=0,46$ & \multicolumn{4}{|l|}{$\mathrm{F}=6,13 ; \mathrm{p}<0,001$} \\
\hline Escalas & Variáveis Explicativas & Beta & $t$ & Sig \\
\hline Sat_Exp_Ac & Opo_Desenvlv & 0,22 & 2,04 & $0,04^{*}$ \\
\hline Hab_Soc & Aut_Exp & 0,19 & 2,16 & $0,03 *$ \\
\hline Mat_Esc_Prof & Com_Realidade & 0,29 & 2,68 & $0,01 *$ \\
\hline
\end{tabular}

Dimensão Instituc do QVA-r

\begin{tabular}{lllll}
\hline R2ajustado & $\mathrm{F}=4,44 ; \mathrm{p}<0,001$ & & \\
\hline Escalas & Varáveis Explicativas & Beta & $t$ & Sig \\
\hline Mat_Esc_Prof & Determinação & $-0,16$ & $-2,23$ & $0,03^{*}$ \\
\hline & Com_Realidade & 0,38 & 3,23 & $0,00^{*}$ \\
\hline
\end{tabular}

\begin{tabular}{lllll}
\hline Vivências Aca dêmicas & & & \\
\hline R2ajustado $=0,49$ & $\mathrm{~F}=6,72 ; \mathrm{p}<0,001$ & & \\
\hline Escalas & Variáveis Explicativas & Beta & $t$ & Sig \\
\hline Sat_Exp_Aca & Curso & 0,28 & 2,26 & $0,01^{*}$ \\
Ma_Esc_Prof & Determinação & $-0,15$ & $-2,22$ & $0,03^{*}$ \\
& Aut_Conhec & 0,27 & 2,54 & $0,01^{*}$ \\
\hline
\end{tabular}


$\mathrm{Na}$ Tabela 2, as variáveis Expectativas Acadêmicas, Satisfação com Experiências Acadêmicas e Habilidades Sociais impactaram positivamente explicando $48 \%$ da variável critério.

\section{Tabela 2}

Regressão Linear Múltipla dos escores gerais das variáveis explicativas: Experiência Acadêmica, Satisfação com a Experiência Acadêmica e Habilidades Sociais na variável critério Vivencias Acadêmicas

\begin{tabular}{llll}
\hline Vivências Acadêmicas & & & \\
\hline R2ajustado $=0,48$ & $\mathrm{~F}=29,35 ; \mathrm{p}<0,001$ & & Sig \\
\hline Variáveis Explicativas & Beta & $t$ & $0,00^{*}$ \\
\hline Exp_Acad & 0,20 & 2,99 & $0,00^{*}$ \\
Sat_Exp_Acad & 0,35 & 5,51 & $0,00^{*}$ \\
Hab_Soc & 0,38 & 5,79 & \\
\hline
\end{tabular}

\section{Discussão}

Para o fator Determinação da EMEP, na dimensão pessoal, que diz respeito ao grau de segurança e decisão ante a escolha profissional (Neiva, 1995), os resultados mostraram que quanto menos seguro em relação à escolha realizada, menos bem estar físico e psicológico o estudante apresenta, podendo inclusive ter problemas de saúde, ou seja, o grau de insegurança e indecisão quanto à escolha do curso causa um impacto negativo no bem estar físico e psicológico do jovem universitário nos doze primeiros meses de curso.

Estudos apontam a indecisão quanto à escolha de uma profissão como um conceito multidimensional e, em se tratando da fase da adolescência, é um estado esperado, frente às questões da escolha de carreira (Osipow, 1999). Entretanto, mesmo sendo esperado, estudos realizados no Rio Grande do Sul, indicaram que a indecisão se correlaciona com as dificuldades, tais como: maior dependência dos pais, retraimento e dificuldade em explorar novas possibilidades profissionais (Magalhães et al., 1998) e a indicadores de ansiedade e depressão (Frischenbruder, 1999).

Ao entrarem na universidade, os estudantes trazem expectativas sobre o que vão encontrar nesse novo ambiente, e a desilusão relacionada ao que de fato vivenciam faz com que os alunos não desenvolvam as relações interpessoais que esperavam. Ao se tratar de alunos do primeiro ano, isso pode ser explicado por uma possível visão irrealista sobre os 
desafios que estariam experimentando nesse novo contexto, fazendo com que suas expectativas iniciais não fossem condizentes com a realidade a ser enfrentada na universidade (Araújo \& Almeida, 2015; Soares et al, 2018).

Ainda na dimensão Interpessoal, os alunos que demostram satisfação com o curso conseguem estabelecer no contexto universitário boas relações com colegas, professores, desmonstrando dessa forma que, estar satisfeito com o curso favorece a abertura para o estabelecimento de relações sociais na universidade, favorecendo o aprofundamento no conteúdo, possibilitando assim, uma melhor qualidade de formação (Suehiro \& Andrade, 2018).

O impacto da satisfação com o Curso na Carreira indica que o estudante que se percebe apto para lidar com diferentes tarefas da futura profissão compreende a importância de se planejar, de ter conhecimento de si e do ambiente e ser capaz de tomar decisões enriquecedoras para a futura carreira, tem condições de lidar com as exigências próprias do Ensino Superior. A satisfação com o curso tem associação com as informações prévias sobre a graduação e a instituição no que concerne aos recursos e opotunidades acadêmicas oferecidas aos estudantes. Santos et al. (2013) destacam que as atividades extra-curriculares colaboram para a formação pessoal e profissional e contribuem com a adaptação do estudante ao curso e à futura carreira.

Em relação ao fator Oportunidade de Desenvolvimento da ESEA, alunos que aproveitam melhor as oportunidades de desenvolvimento oferecidas pela universidade como palestras, programas de estágio, atividades disponibilizadas para o seu desenvolvimento profissional e serviços de apoio ao estudante, apresentam bons hábitos de estudo e conseguem administrar seu tempo de modo satisfatório. De fato, em estudo realizado por Santos et al. (2013), foi identificado um elevado índice de satisfação com as vivências acadêmicas, tendo sido encontrada uma correlação positiva e significativa entre as dimensões Estudo do QVA-r e Oportunidade de Desenvolvimento do ESEA. Para os autores, essa relação é fundamental para a qualidade das vivências e, por consequência, da adaptação acadêmica, pois, por um lado os estudantes modificam o ambiente a partir de suas percepções, escolhas e ações, e por outro, o ambiente impacta nos alunos em decorrência das experiências possibilitadas, das normas e oportunidades oferecidas.

No fator Autoexposição a Desconhecidos e Situações Novas do IHS, alunos que têm boa desenvoltura com pessoas desconhecidas diante de situações novas conseguem planejar sua rotina de estudos e se preparam melhor para as avaliações. Bartholomeu et al. (2008) afirmam que universitários mais competentes socialmente tendem a ser mais atenciosos, 
compreensivos, empáticos e agradáveis com os colegas e os demais indivíduos que compõem a comunidade acadêmica.

No que diz respeito ao fator Conhecimento da Realidade Profissional da EMEP, universitários que conhecem mais sobre as características das profissões e as exigências do mercado de trabalho demonstram boa capacidade de estudar e são eficientes na preparação para as provas. Lorga (2017) corrobora essa ideia ao sinalizar que os estudantes não estão apenas preocupados com suas aprovações, mas também com informações relevantes acerca do seu futuro profissional e com os aspectos que podem interferir na sua tomada de decisão.

Segundo os resultados apontados no fator Curso da ESEA, pode-se pensar que o estudante, ao ingressar na universidade, vivencia experiências favoráveis na instituição quanto mais ele encontra-se satisfeito com o curso, gerando assim maior probabilidade de satisfação acadêmica. $\mathrm{O}$ resultado positivo da satisfação nos estudantes do primeiro ano também pode ser explicado considerando que os alunos reconhecem as dificuldades enfrentadas como algo natural e esperado no processo de adaptação, permitindo-se maior tolerância (Bardagi \& Hutz, 2010). Corroborando os resultados deste estudo, Schleich et al. (2006) avaliaram a satisfação acadêmica entre estudantes ingressantes e concluintes de uma universidade paulista e encontraram maior satisfação entre os alunos ingressantes, especialmente nos domínios de satisfação com o curso e percepção de oportunidades de crescimento. Santos et al. (2013) consideram que, se por um lado, a satisfação mostra-se associada à integração ao Ensino Superior, pois interfere no envolvimento do aluno com a instituição e com o curso e na sua decisão de permanência; por outro lado, estabelece a própria experiência de integração, abrangendo as vivências pessoais, acadêmicas e sociais.

Em relação ao fator Autoconhecimento, quanto mais conhecimento sobre si o aluno demonstra, possivelmente mais adaptado à universidade ele está. $\mathrm{O}$ autoconhecimento foi evidenciado no estudo realizado por Moura e Menezes (2004) com a principal dificuldade de 75\% dos universitários inscritos em um programa de reorientação profissional. Os participantes mostravam-se confusos, uns por não identificarem seus interesses, outros por conhecê-los mas não saberem como analisá-los para compor sua decisão. Alguns estudos apontam a relação do autoconhecimento com outras variáveis: escolha profissional madura, ajustada, pressupõe capacidade de adaptação, de discriminação, análise e integração de conhecimentos sobre si e sobre a realidade profissional e aprendizagem do processo de tomada de decisão (Neiva, 1995). No que diz respeito ao fator determinação da EMEP na dimensão pessoal, quanto mais inseguros os alunos se sentem em relação à escolha profissional realizada, menos adaptados eles se encontram. Nogueira et al. (2020), em estudo 
com estudantes de Psicologia, apontam que a maturidade para a escolha profissional está associada positivamente às vivências na universidade e à carreira por meio do fator determinação da EMEP. Assim, o estudante satisfeito com a escolha do curso e da instituição procura empenhar-se para superar as dificuldades encontradas nas questões de estudo e os desafios institucionais da vida acadêmica.

É esperado que antes de ingressar no Ensino Superior o aluno tenha informações sobre a carreira que pretende iniciar, assim como o que a instituição de ingresso tem a oferecer para a formação almejada. As expectativas iniciais sobre o curso e a carreira (Soares et al, 2018) e a satisfação na escolha profissional podem explicar a incidência de permanência ou evasão observada logo nos primeiros períodos das graduações (Santos et al., 2103). A permanência e a formação com qualidade têm possibilitado pesquisas e intervenções institucionais no acolhimento ao aluno, considerando as diversidades de experiências trazidas e a busca pelo sucesso acadêmico almejado (Bisinoto et al., 2016).

\section{Considerações Finais}

Ao considerar o contexto dos estudantes do primeiro ano do curso de Psicologia de instituições públicas e privadas do estado do Rio de Janeiro, este estudo teve por objetivo verificar se as variáveis explicativas que constituem o modelo teórico testado explicam a variável critério adaptação acadêmica. Antecedendo o ingresso à universidade, ações individuais ou programas institucionais que forneçam informações e orientações no que concerne a escolha da graduação muito favoreceriam os fatores que explicaram a adaptação acadêmica, como o autoconhecimento, a determinação, as expectativas acadêmicas, o conhecimento da realidade e a satisfação com a graduação. A implementação de ações preventivas supostamente inibe sentimentos de insegurança e insatisfação em relação às escolhas profissionais, assegurando o compromisso e o empenho com hábitos de estudo que propiciam o processo de integração e de aprendizagem. Além disso, estudantes ingressantes que apresentam boas habilidades para lidarem com pessoas desconhecidas em contextos diversificados tendem a se adaptar melhor ao ambiente universitário.

Em termos de limitações, o presente estudo poderia ter abrangido uma amostra que contasse com diferentes graduações, de outras localidades da federação e tipo de instituição. Pesquisas futuras sobre adaptação acadêmica de estudantes de Psicologia podem considerar dados sociométricos, como sexo, idade e nível socioeconômico. 


\section{Referências}

Almeida, L. S., Ferreira, J. A. G., \& Soares, A. P. (2002). Questionário de Vivências Acadêmicas (QVA-r): Avaliação do ajustamento dos estudantes universitários. Avaliação Psicológica, 1(2), 81-93. Recuperado de http://pepsic.bvsalud.org/scielo.php?script=sci_arttext\&pid=S167704712002000200002

Araújo, A. M., \& Almeida, L. S. (2015). Adaptação ao Ensino Superior: O papel do moderador das expectativas adacêmicas. Lumen Educare, 1(1), 13-32. doi: 10.19141/2447-5432/lumen.v1.n1.p.13-32

Bardagi, M. P., \& Hutz, C. S. (2010). Satisfação da vida, comprometimento com a carreira e exploração vocacional em estudantes universitários. Arquivos Brasileiros de Psicologia, 62(1), 159-170. Recuperado de http://pepsic.bvsalud.org/scielo.php?script=sci_arttext\&pid=S180952672010000100016\#: :text=Em\%20universit\%C3\%A1rios\%2C\%20avaliar\%20o\%2 0n\%C3\%ADvel,o\%20curso\%20e\%20a\%20profiss\%C3\%A3o

Bartholomeu, D., Nunes, C. H. S., \& Machado, A. A. (2008). Traços de personalidade e habilidades sociais em universitários. Psico-USF, 13(1), 41-50. doi: 10.1590/S141382712008000100006

Bisonoto, C., Rabelo, M. L., Marinho-Araujo, C., \& Fleith, D. D. (2016). Expectativas acadêmicas dos ingressantes da Universidade de Brasília: Indicadores para uma política de acolhimento. In L. S. Almeida \& R. V. Castro (Orgs.), Ser estudantes no ensino superior: $O$ caso dos estudantes do $1^{o}$. Ano (pp. 15-31). Portugal, Braga: Universidade do Minho.

Bolsoni-Silva, A. T., Loureiro, S. R., \& Oliveira, M. C. F. A. (2010). Caracterização das habilidades sociais de universitários. Contextos Clínicos, 3(1), 62-75. Recuperado de http://pepsic.bvsalud.org/scielo.php?script=sci_arttext\&pid=S198334822010000100007\#: :text=As\%20Habilidades\%20Sociais\%20(HS)\%2C,de\%20soc ializa\%C3\%A7\%C3\%A3o\%20saud\%C3\%A1vel\%20e\%20satisfat\%C3\%B3rio

Del Prette, A., \& Del Prette, Z. A. P. (Orgs.). (2017a). Habilidades sociais intervenções efetivas em grupo (2a ed.). São Paulo: Pearson.

Del Prette, A., \& Del Prette, Z. A. P. (2017b). Competência Social e Habilidades Sociais: Manual teórico-prático. Petropólis, RJ: Vozes. 
Del Prette, Z. A. P., \& Del Prette, A. (2001). Psicologias das habilidades sociais: Terapia e educação. Petropólis, RJ: Vozes.

Fernández, M. F. P., Araújo, A. M., Vacas, C. T., Almeida, L. S., \& González, M. R. S. (2017). Predictors of students' adjustment during transition to university in Spain. Psicothema, 29(1), 67-72. doi: 10.7334/psicothema2016.40

Freitas, H. C. N. M., Raposo, N. A.V. R., \& Almeida S. A. (2007). Adaptação do estudante ao ensino superior e rendimento académico: Um estudo com estudantes do primeiro ano de enfermagem. Revista Portuguesa de Pedagogia, 41(1), 179-188. doi: 10.14195/1647-8614_41-1_10

Frischenbrüder, S. L. (1999). O desenvolvimento vocacional na adolescência: Autoconceito e comportamento exploratório (Dissertação de Mestrado). Pontifícia Universidade Católica do Rio Grande do Sul, Porto Alegre, Brasil.

Granado, J. I. F., Santos, A. A. A., Soares, A. P., \& Guisande, M. A. (2005). Integração Académica de estudantes universitários: Contributos para a adaptação e validação do QVA-r no Brasil. Psicologia e Educação, 4(2), 31-41. Recuperado de http://hdl.handle.net/1822/12089

Lehman, Y. P. (2014). University students in crisis: University dropout and professional reselection. Estudos de Psicologia, 31(1), 45-53. doi: 10.1590/0103166X2014000100005

Lorga, J. M. L. (2017). Orientação profissional: A maturidade para escolha profissional entre estudantes da $3^{\text {a }}$ série do Ensino Médio. Revista Ciência Amazônica, 1(2), 1-19. Recuperado de http://www.periodicos.ulbra.br/index.php/amazonida/article/view/2978 Magalhães, M. O., Lassance, M. C. P., \& Gomes, W. B. (1998). Escolha vocacional em adolescentes. In W. B. Gomes (Org.) Fenomenologia e pesquisa em psicologia (pp.161-196). Porto Alegre: EDUFRGS.

Marinho-Araujo, C. M., Souza Fleith, D., Almeida, L. S., Bisinoto, C., \& Rabelo, M. L. (2015). Adaptação da escala expectativas acadêmicas de estudantes ingressantes na educação superior. Avaliaçao Psicologica: Interamerican Journal of Psychological Assessment, $\quad 14(1), \quad 133-141 . \quad$ Recuperado de http://pepsic.bvsalud.org/scielo.php?script=sci_arttext\&pid=S167704712015000100016

Matta, C. M. B., Lebrão, S. M. G., \& Heleno, M. G. V. (2017). Adaptação, rendimento, evasão e vivências acadêmicas no ensino superior: Revisão da literatura. Psicologia Escolar e Educacional, 21(3), 583-591. doi: 10.1590/2175-3539/2017/0213111118 
Moura, C. B., \& Menezes, M. V. (2004). Mudando de opinião: Análise de um grupo de pessoas em condição de re-escolha profissional. Revista Brasileira de Orientação $\begin{array}{llll}\text { Profissional, } & 5(1), & \text { 29-45. }\end{array}$ http://pepsic.bvsalud.org/scielo.php?script=sci_arttext\&pid=S167933902004000100004\#: :text=Como\%20encontrado\%20na\%20pesquisa\%2C\%20os,u ma\%20re\%2Descolha\%20mais\%20segura

Neiva, K. M. C. (1995). Entendendo a orientação profissional. São Paulo: Paulus.

Neiva, M.M.C. (2014). Escala de maturidade para escolha profissional (2a ed.). São Paulo: Vetor.

Nogueira, C. C., Soares, A. B., Monteiro, M. C. L., \& Medeiros, H. C. P. (2020). Habilidades sociais e expectativas acadêmicas em universitários: Um estudo comparativo entre estudantes de enfermagem e de outras áreas da saúde. Estudos e Pesquisas em Psicologia, 1(1), 99-118. doi: 10.12957/epp.2020.50792

Oliveira, R. E. C. D., \& Morais, A. D. (2015). Vivências acadêmicas e adaptação de estudantes de uma universidade pública federal do Estado do Paraná. Revista de Educação Pública, 24(57), 547-568. doi: 10.29286/rep.v24i57.1796

Oliveira, E. P., Merino, M. D., Privado, J., \& Almeida, L. S. (2017). Funcionamento psicológico positivo numa amostra portuguesa de estudantes. Revista de Estudios e Investigación en Psicología y Educación, 7, 1-5. doi: 10.17979/reipe.2017.0.07.2733

Osipow, S. H. (1999). Assessing career indecision. Journal of Vocational Behavior, 55(1), 147-154. doi: 10.1006/jvbe.1999.1704

Santos, A. A. A., Polydoro, S. A. J., Scortegagna, S. A., \& Linden, M. S. S. (2013). Integração ao ensino superior e satisfação acadêmica em universitários. Psicologia, Ciência e Profissão, 33(4), 780-793. doi: 10.1590/S1414-98932013000400002

Schleich, A, L. R., Polydoro, S. A. J., \& Santos, A. A. (2006). Escala de Satisfação com a experiência acadêmica de estudantes do Ensino Superior. Avaliação Psicológica, 5(1) $11-20$.

Recuperado de http://pepsic.bvsalud.org/scielo.php?script=sci_arttext\&pid=S167704712006000100003

Soares, A. P., Almeida, L. S., Diniz, A. M., \& Guisande, M. A. (2006). Modelo multidimensional de ajustamento de jovens ao contexto universitário (MMAU): Estudo com estudantes de ciências e tecnologias versus ciências sociais e humanas. Análise Psicológica, 1(24), 15-27. Recuperado de 
Adriana Benevides Soares, Claudio de Almeida Lima, Marcia Cristina Monteiro, Bruno de Alcântara Sousa, Giselle Glória Balbino dos Santos, Isabela da Silva Rodrigues

http://www.scielo.mec.pt/scielo.php?script=sci_arttext\&pid=S0870-

82312006000100003

Soares, A. B., Leme, V. R., Gomes, G., Peçanha, A. P. C. L., Maia, F. A., Lima, C. A., Valadas, S., Almeida, L., \& Araujo, A. (2018). Expectativas acadêmicas de estudantes nos primeiros anos do Ensino Superior. Arquivos Brasileiros de Psicologia, 70(1), 206-223.

Recuperado

de

http://pepsic.bvsalud.org/scielo.php?script=sci_abstract\&pid=S1809-

$52672018000100015 \& \operatorname{lng}=\mathrm{pt} \& n r m=\mathrm{iso} \& \operatorname{lng}=\mathrm{pt}$

Suehiro, A. C. B., \& Andrade, K. S. (2018). Satisfação com a experiência acadêmica: Um estudo com universitários do primeiro ano. Psicologia e Pesquisa, 12(2), 1-10. doi: $10.24879 / 2018001200200147$

Travassos, R., \& Mourão, L. (2018). Lacunas de competências de egressos do curso psicologia na visão dos docentes. Psicologia: Ciência e Profissão, 38(2), 233-248. doi: 10.1590/1982-3703004472016

\section{Endereço para correspondência}

\section{Adriana Benevides Soares}

Rua Marechal Deodoro, 217, Niterói - RJ, Brasil. CEP 24030-060

Endereço eletrônico: adribenevides@gmail.com

\section{Claudio de Almeida Lima}

Rua Marechal Deodoro, 217, Niterói - RJ, Brasil. CEP 24030-060

Endereço eletrônico: limaclaudio74@hotmail.com

\section{Marcia Cristina Monteiro}

Rua Marechal Deodoro, 217, Niterói - RJ, Brasil. CEP 24030-060

Endereço eletrônico: marcialauriapsi@outlook.com

\section{Bruno de Alcântara Sousa}

Rua Marechal Deodoro, 217, Niterói - RJ, Brasil. CEP 24030-060

Endereço eletrônico: linearbruno@gmail.com

\section{Giselle Glória Balbino dos Santos}

Rua Marechal Deodoro, 217, Niterói - RJ, Brasil. CEP 24030-060

Endereço eletrônico: gbsantos25@gmail.com

\section{Isabela da Silva Rodrigues}

Rua Marechal Deodoro, 217, Niterói - RJ, Brasil. CEP 24030-060

Endereço eletrônico: rodrigues.isabelasilva@gmail.com 
Adriana Benevides Soares, Claudio de Almeida Lima, Marcia Cristina Monteiro, Bruno de Alcântara Sousa, Giselle Glória Balbino dos Santos, Isabela da Silva Rodrigues

Recebido em: 22/08/2019

Reformulado em: 05/10/2020

Aceito em: $12 / 10 / 2020$

\section{Notas}

* Doutora em Psicologia pela Universidade de Paris XI, Professora Titular no Programa de Pós-Graduação da Universidade Salgado de Oliveira e Professora Titular na Universidade do Estado do Rio de Janeiro (UERJ).

** Mestre em Psicologia Social pela Universidade Salgado de Oliveira (UNIVERSO).

*** Doutora em Psicologia Social pela Universidade Salgado de Oliveira, Professora Titular no Grupo Universidade Brasil/UNIESP-FDC.

**** Doutorando em Psicologia Social pela Universidade Salgado de Oliveira (UNIVERSO), Professor na Fundação de Apoio à Escola Técnica (FAETEC) e na rede estadual de ensino do Rio de Janeiro.

****** Mestre em Psicologia Social pela Universidade Salgado de Oliveira (Universo), Orientadora Pedagógica na Fundação de Apoio à Escola Técnica (FAETEC).

****** Mestre em Psicologia Social pela Universidade Salgado de Oliveira (Universo).

Financiamento: CNPQ - bolsa de produtividade para a primeira autora.

Este artigo de revista Estudos e Pesquisas em Psicologia é licenciado sob uma Licença Creative Commons Atribuição-Não Comercial 3.0 Não Adaptada. 